\title{
D isección espontánea de la arteria renal e infarto renal: Dos casos clínicos
}

\author{
Francisco Borjaa, Renato González ${ }^{1}$, Nicolás Rodrígueza \\ Marcela U rsu², Cristián Varela ${ }^{3}$, Antonio Vukusich².
}

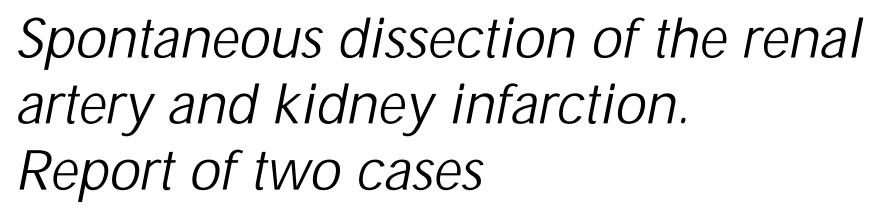

We report two previously healthy males aged 33 and 37 years, presenting with severe pain in the right and left part of the abdomen, respectively. An abdominal CT scan showed in both a kidney infarction. An angio-CAT scan showed changes compatible with a fibromuscular dysplasia in the renal arterial wall. An angiography showed an intimal tear or complex dissection flap in both cases. Both had a satisfactory evolution with conservative treatment. The relationship between fibromuscular dysplasia and spontaneous dissection of the renal artery is discussed (Rev Méd Chile 2008; 136: 1183-7).

(Key words: Angiography; Kidney cortex necrosis; Renal artery)

Recibido el 11 de septiembre, 2007. Aceptado el 9 de enero, 2008.

${ }^{1}$ Servicios de Hemodinamia, ${ }^{2}$ Nefrología y de ${ }^{3}$ Radiología, Clínica Dávila. Santiago de Chile. anterno de Medicina, Facultad de Medicina, Universidad de Los Andes. Santiago de Chile.

L a disección espontánea de la arteria renal (DEAR) es una causa infrecuente de dolor abdominal, por lo que su diagnóstico suele aplazarse al confundirse con otros cuadros de similares características ${ }^{1}$.

Las arterias renales son los vasos periféricos más afectados por disección primaria, debiéndose ésta a lesiones iatrogénicas, trauma o arteriopatía como fibrodisplasia, enfermedad ateroesclerótica o del tejido conectivo.

La DEAR se ve más frecuentemente en hombres sanos en la cuarta o quinta década de vida, presentándose de forma aguda pudiendo resultar en la pérdida de parénquima renal e hipertensión arterial severa ${ }^{2}$.

Para su diagnóstico precoz, es fundamental la sospecha clínica y la utilización de técnicas radioló-

Correspondencia a: Francisco Borja Consigliere. Alsacia 57, Dpto. 911, Las Condes, Santiago. Código Postal: 7550028. Teléfono: +56-2-2285942. E mail: fborjac@hotmail.com gicas adecuadas, hoy en día angioTC, angiorresonancia nuclear magnética (angioRNM) y angiografía.

\section{CASO CLÍNICO 1}

Hombre de 33 años con antecedentes de ser fumador (20 cig/día) y un episodio de urolitiasis a los 27 años. Consultó por cuadro de dolor intenso en flanco derecho, de inicio súbito, sin irradiación, asociado a náuseas y vómito en una ocasión que cede espontáneamente. El cuadro se repitió $5 \mathrm{~h}$ después, siendo de similares características al anterior.

No presentó fiebre y al examen físico destacaba una presión arterial de 140/82 $\mathrm{mmHg}$ y dolor a la palpación en fosa ilíaca derecha (FID).

Dentro de los exámenes de laboratorio se encontró orina completa sin hematuria, hemograma con leucocitosis $\left(17.200 \mathrm{x} \mathrm{mm}^{3}\right)$, creatininemia de $1,2 \mathrm{mg} / \mathrm{dl}$ y LDH de $1.457 \mathrm{UI} / \mathrm{l}$. 
Se realizó pieloTC que resultó ser negativa para urolitiasis, prosiguiéndose con TC de abdomen y pelvis, en la cual se observó una extensa área hipodensa de aspecto cuneiforme en la cara posterior del parénquima renal derecho, compatible con infarto parcial renal derecho.

Se hospitalizó al paciente. Se tomaron exámenes para trombofilia, cuyos resultados fueron normales, previo al inicio de anticoagulación (Fragmin ${ }^{\circledR}$ y Neosintrón ${ }^{\circledR}$ ). Además se realizó ecocardiografía transesofágica que resultó normal y un angioTC que reveló la presencia de una arteria renal derecha dominante (AD) y otra polar de menor calibre con trayecto normal, e imagen compatible con alteración de la pared arterial con infarto renal (Figura 1).

Se prosiguió con estudio angiográfico, el cual reveló una aortografía normal, alteración en la perfusión del polo superior, cara anterior y posterior del riñón derecho, sin compromiso de la perfusión del polo inferior dado por la arteria polar secundaria. La arteria renal dominante se observó irregular, dilatada a partir de su tercio medio que además presentó un flap de disección que comprometió el origen de las ramas interlobares, limitando así el flujo distal. El flap de disección fue descrito como de alta complejidad sin posibilidades de maniobras endovasculares (Figura 2).

Dos días después presentó nuevo episodio de dolor en flanco derecho. Nuevo angioTC mostró una clara progresión del infarto renal en los 2/3 superiores del riñón derecho (Figura 3).

El paciente evolucionó con presiones arteriales en el rango de 160/100 $\mathrm{mmHg}$ y sin deterioro de la función renal (creatininemia $1,2 \mathrm{mg} / \mathrm{dl}$ ). Fue dado de alta con terapia antihipertensiva (Blox ${ }^{\circledR} 4$ $\mathrm{mg} /$ día) en buenas condiciones generales para su manejo ambulatorio. En control ambulatorio a los dos y diecisiete días posterior al alta se encontró al paciente asintomático, normotenso y con el resto del examen físico sin hallazgos patológicos.

\section{CASO CLÍNICO 2}

Hombre de 37 años previamente sano que consultó por dolor abdominal intenso inicialmente de tipo cólico en hipocondrio izquierdo que luego pasó a ser constante en flanco izquierdo; sin irradiación y sin otro síntoma asociado. Al examen se encontraba normotenso y presentaba leve distensión abdominal. No obstante al tratamiento analgésico, persistió sintomático por lo que se realizó TC abdomenpelvis, el cual reveló un área hipodensa de aspecto cuneiforme de la corteza del polo inferior del riñón izquierdo compatible con un infarto renal.

Se realizó una ecocardiografía que descartó la presencia de trombos.

Se prosiguió el estudio con angioTC, donde la aorta se observó normal y el segmento distal de la arteria renal izquierda y su rama anterior aparecieron alterados, dando imágenes compatibles con fibrodisplasia arterial e infarto renal (Figura 4).

Posteriormente se realizó una angiografía, en la cual destacaba una arteria renal izquierda de aspecto fibrodisplásico con un flap de disección en su rama interlobar inferior. No se realizaron maniobras endovasculares (Figura 5).

Dentro de los exámenes pedidos se tomaron pruebas para trombofilia que resultaron normales y se verificó una buena función renal (creatininemia de 1,0 mg/dl). El paciente evolucionó normotenso dándose de alta para su manejo ambulatorio. Sin embargo, el paciente no asistió a control.

\section{DisCUSIÓN}

La disección espontánea de la arteria renal (DEAR) es una entidad poco frecuente, no existen grandes series publicadas al respecto ni tampoco consensos concernientes al manejo más adecuado.

La mayoría de las disecciones provocan una oclusión arterial, aunque también puede presentarse una lesión degenerativa aneurismática de la arteria. En cuanto a la etiología de la DEAR, se ha visto asociada a diversas patologías que comprometen la pared arterial, incluidas la mencionada displasia fibromuscular, una entidad aún poco conocida, que en la mayoría de los casos afecta la capa media de la arteria renal. La displasia fibromuscular consiste en una lesión intrínseca de la pared arterial sin componentes inflamatorios ni ateroescleróticos. Puede afectar diversos territorios, pero predomina en las arterias renales (60\% a $75 \%$ de los casos) siendo en 35\% bilateral. En cuanto a su patogenia se han propuesto una variedad de factores genéticos, mecánicos y hormonales. Sin 


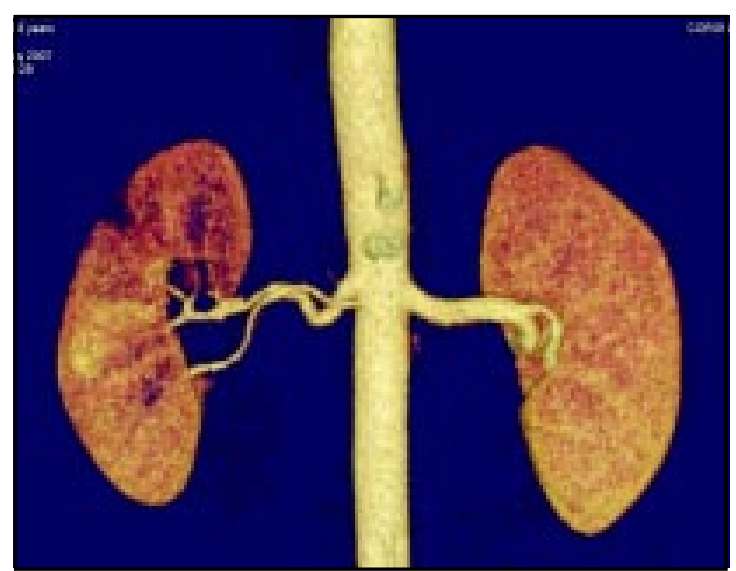

Figura 1. AngioTC renal que muestra la presencia de una arteria renal derecha dominante (AD) y otra polar de menor calibre con trayecto normal. La AD con franca disminución de su calibre en $1 / 2$ distal con presencia de imagen hipodensa, alargada, de 1,6 cm de longitud, compatible con enfermedad de pared arterial. También se observa un gran segmento cortical posterior de riñón derecho con defecto de impregnación, hipodenso y cuneiforme compatible con un infarto renal.

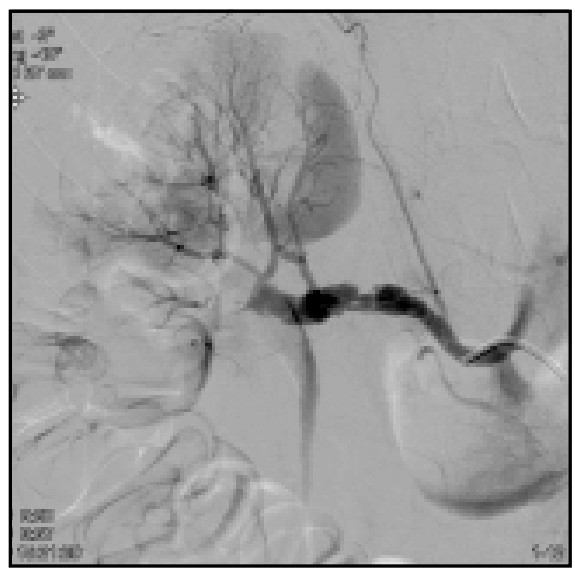

Figura 2. Angiografía renal derecha. El riñón se encuentra hipoperfundido en sus 2/3 superiores, mientras que una arteria polar irriga el tercio inferior. La arteria renal derecha es irregular, dilatada a partir del tercio medio, comprometiéndose el origen de las ramas interlobares del riñón por un trozo de disección complejo que limita el flujo.

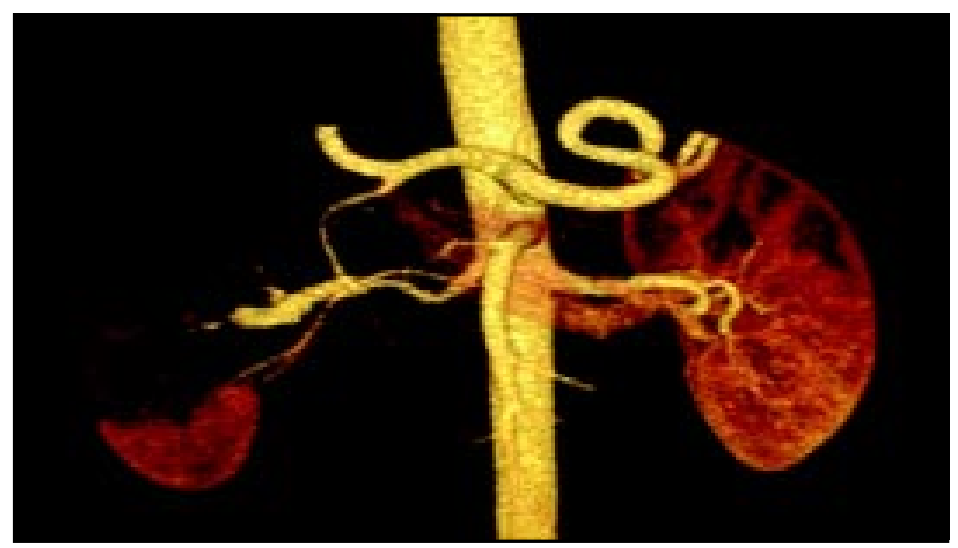

Figura 3. AngioTC renal de seguimiento muestra una clara progresión del infarto renal en los 2/3 superiores del riñón derecho.

Figura 4. AngioTC. La aorta se observa normal, el segmento distal de la arteria renal izquierda y su rama anterior aparecen dilatados, de bordes imegulares, con aspecto armosariado y leve engrosamiento mural. Estos mismos hallazgos se observan en menor magnitud en las ramas hiliares. Además se observa en el aspecto lateral del polo inferior del riñon izquierdo un segmento que no se perfunde, de bordes cuneiformes compatibles con un infarto.

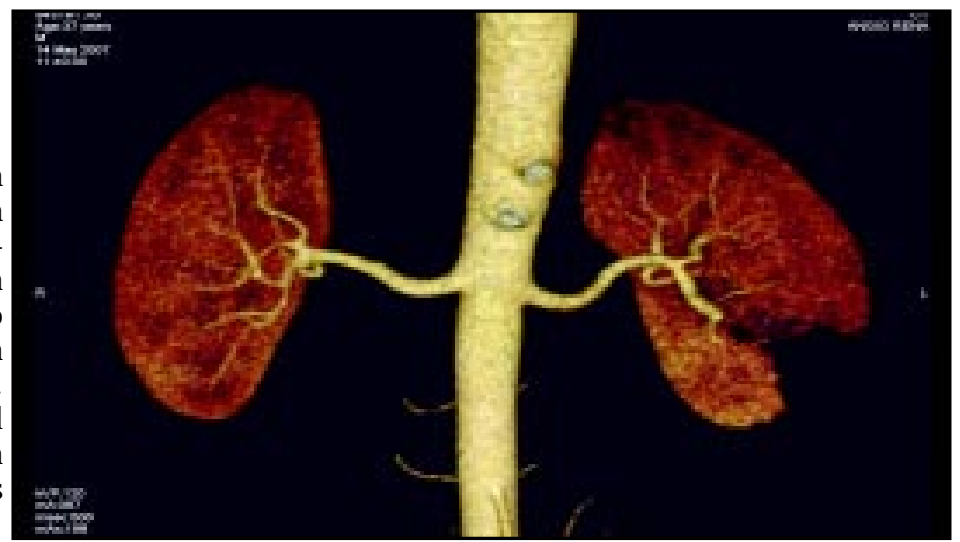




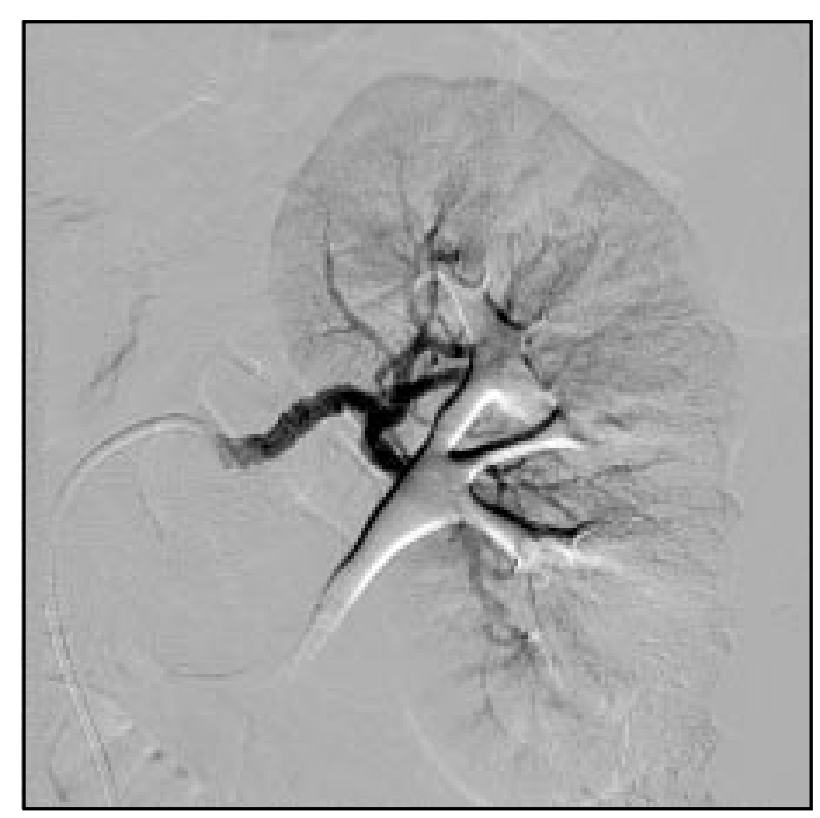

Figura 5. Angiografía renal izquierda. Destaca una arteria renal de aspecto fibrodisplásico cuya rama interlobar inferior presenta una disección espontánea que compromete ramas secundarias.

embargo, la causa de la displasia fibromuscular permanece desconocida. Como factores de riesgo, se identifican el tabaquismo, la hipertensión arterial $\mathrm{y}$ el tener pariente de primer grado con esta condición. Dentro de sus complicaciones se encuentran la progresión focal de la lesión, estenosis arterial, hipertensión arterial renovascular, aneurismas murales y la disección espontánea de la arteria $^{3}$. Otras causas de DEAR son la ateroesclerosis severa, síndrome de Marfán, hipertensión maligna y el síndrome de Ehlers-Danlos ${ }^{4}$.

En el paciente que se queja de dolor en flanco, la disección de la arteria renal debiera estar considerada dentro de los diagnósticos diferenciales. Hoy en día existen exámenes radiológicos no invasivos y cada vez más accesibles que nos permiten lograr diagnósticos precoces y adecuados. No obstante, la angiografía sigue siendo el estudio que otorga la confirmación diagnóstica, además de contar con ciertas posibilidades terapéuticas.

Las estrategias terapéuticas para la DEAR deben ser planeadas de manera cuidadosa depen- diendo de las posibilidades técnicas y de los objetivos planteados. Se han propuesto tanto tratamientos quirúrgicos, endovasculares, como médicos para conservar la función renal y evitar la hipertensión ${ }^{1,5-9}$. Sin embargo, el diagnóstico suele ser tardío y no existe consenso respecto a la indicación de cada modalidad.

Algunos pacientes han tenido buen resultado con un manejo conservador $1,2,4,5,12$, mientras que otros han debido ser nefrectomizados para controlar la hipertensión ${ }^{6,9-11}$.

Actualmente existen antecedentes en la literatura que apoyan intentar un manejo quirúngico o endovascular sólo en DEAR diagnosticadas precozmente en riñones de tamaño no menor a $9,5-10 \mathrm{~cm}$ de longitud, debido a que riñones de tamaños menores tienen un pronóstico funcional pobre ${ }^{9,10,12}$. En estos casos estaría indicado un manejo conservador con un adecuado control de la presión arterial.

Finalmente, la nefrectomía se reserva para los casos en los que coexisten un riñón pequeño e hipertensión inmanejable ${ }^{2}$. 


\section{REFERENCIAS}

1. Ramamoorthy SL, Vasquez JC, Taft PM, Mcginn RF, Hye RJ. Nonoperative management of acute spontaneous renal artery dissection. Ann Vasc Surg 2002; 16: $157-62$.

2. Ando T, Ohno H, Hirata Y, Emoto A, Ogata S, Mimata $\mathrm{H}$. Spontaneous recovery from renal infarction resulting from renal artery dissection. Int J Urol 2005; 12: 405-8.

3. Stovut D, Oun J. Current Concepts Fibromuscular Dysplasia. N Engl J Med 2004; 350; 18: 1862-71.

4. Hatsuki H, OHichi I, Keni M, Yoshiaki Y, Toshio S. A case of spontaneous recanalization following renal infarction. Acta Urol Jpn 1993; 39: 159-62.

5. Amir A, Donald FM, Peter B. Renal artery dissection causing renal infarction otherwise healthy men. Am J Kidney Dis 1997; 30: 851-5.

6. Van Rooden CJ, Van Baalen JM, Van Bochel JH. Spontaneous dissection of renal artery: long-term results of extracorporeal reconstruction and autotransplantation. J Vasc Surg 2003; 38: 116-22.
7. Dobrilovic N, Bennett S, Smith C, Edwards J, Luchette FA. Traumatic renal artery dissection identified with dynamic helical computed tomography. J Vasc Surg 2001; 34: 562-4.

8. Sharples E J, Sobeh M, Matson M, Yaqoob MM. Renal artery dissection after blunt abdominal trauma: a rare cause of acute cortical necrosis. Am J Kidney Dis 2002; 40: E11.

9. Barbara TM, Lutz R, Tomas P. Surgical treatment of renal artery dissection in 25 patients: indications and results. J Vasc Surg 2003; 37: 761-8.

10. LaWrie GM, Morris GC, DebaKey ME. Long-term result of treatment of the totally occluded renal artery in forty patients with renovascular hypertension. Surgery 1980; 88: 753-9.

11. Scott AS, Ernest EH, Andrew CN, Thomas M. Surgical treatment for isolated dissection of the renal artery. J Urol 1990; 144: 233-7.

12. Mori H, Hayashi $K$, Tasaki $T$, Hori $T$, Yamasaki $T$, Амамото Y. Spontaneous resolution of bilateral renal artery dissection: a case report. J Urol 1986; 135: $114-6$ 